\title{
Demonstration of the IdeaBoard Interface and Applications
}

\author{
Tsuyoshi Oguni, Takashi Yoshino and Masaki Nakagawa
}

\author{
Dept. of Computer Science \\ Tokyo Univ. of Agri. \& Tech. \\ Naka-cho 2-24-16, Koganei, Tokyo, 184, Japan \\ oguni@hands.ei.tuat.ac.jp
}

\begin{abstract}
This video demonstrates a new style of human interface and several applications on an interactive dynamic whiteboard (Nakagawa et al 1997). Its design issues have been how to realize easy and natural operation to the large board from an arbitrary standing position of its user with a reasonable amount of hand movement, avoidance of unreliable gesture commands as well as smooth and natural extension of the current desktop GUI.
\end{abstract}

KEYWORDS pen interface, handwriting recognition, electronic whiteboard, direct pointing, GUI

\section{INTRODUCTION}

A white/black board is one of the devices which are not fully computerized although it may open the new way of presentation and lecture. We decided to develop a system to combine the merit of the white/black board and that of computer processing as a part of our pen interface research.

The Liveboard by XEROX is well-known as such a system (Elrod et al. 1992, Pedersen et al. 1993, Weiser 1991, 1993). Our system mainly differs from the Liveboard in the following respects.

Firstly, the Liveboard and especially its applications developed at XEROX PARC avoid handwriting recognition except that for gestures (Pedersen 1993). On the other hand, we employ handwriting recognition to provide the power of computing to our behaviors of handwriting. However, we are not positive about employing gestures because of the reasons describe below.

One of the reasons that the Liveboard has employed gestures would be that a simple expansion of the desktop GUI to the board size requires the user to push top left button as well as bottom right buttons, with the result that the user's body often covers the board. We try to find a different solution.

Secondly, the resolution and brightness of the Liveboard seems unsatisfactory. Enlarging a computer display and sharing it by participants make collaboration easier by itself. To be used as an electronic whiteboard, however, brightness and resolution are very important.

We decided to use a temporary hardware to develop interface and applications while making a new hardware. We call our system "IdeaBoard" (Interactive, Dynamic, Electronic Assistant Board).

The basic concept of the IdeaBoard is not the enlargement of the computer display, but the coupling of the merits of a whiteboard with those of computer processing. This requires the very high resolution of a large display (more than $1600 \times 1200$ resolution of the 70 -inch size) and sufficient brightness so that the students can watch the teacher's face as well as the contents on the board in a well-lighted room. Moreover, new human interface design as well as

Human-Computer Interaction: INTERACT'97 S. Howard, J. Hammond \& G. Lindgaard (editors) Published by Chapman \& Hall OIFIP 1997 
applications must be developed which inherit the conventional and familiar interfaces from a white/black board while exploiting the dynamic processing of contents by computing.

\section{GESTURES CONSIDERED HARMFUL}

Gestures have been an attraction of pen interfaces. The Liveboard has also employed gestures, but we are reluctant to employ gestures because of the following reasons:

\section{Pattern recognition problem}

Gestures have simple shapes and simple shapes are hard for machines to recognize. Moreover, there is little context to augment gesture recognition.

2. Human interface problem

Design of gestures is very difficult. Often, gestures are difficult to remember and easy to forget. Moreover, mode change for inputting gestures is troublesome and easy to forget. Misrecognition of gestures or forgotten mode change yields an abrupt and unexpected result and that must be canceled. They not only interrupt lectures or presentations very badly but also shock the teacher or presenter.

Because of these problems, users fear that gestures might be misinterpreted and may hesitate to use gestures.

Therefore, we avoid the human interface being dependent on gestures. Nevertheless, we do not avoid pattern recognition. Pattern recognition can be more useful for contents rather than for commands.

\section{IdeaBoard USER INTERFACE}

If we just expand the desktop GUI to the whiteboard size, a teacher has to move from side to side, stretch hands from button to button, which not only makes the teacher practice gymnastics but often hides the board from the students. To operate the IdeaBoard without crossing the surface, the elements of the desktop GUI should be examined and modified so that it can be operated from an arbitrary standing position.

Considering the body movement to operate the IdeaBoard, if it is too large, it is hard for the user as well as it hides the board, but if it is too minute, it is again difficult for the standing user. We think that body size movement with direct pointing and manipulation is not only appealing to the audience but also navigates their focus of attention.

To keep pushing a marker horizontally at one point on the vertical board is hard for a person. Our hands become tired in trying to remain steady. Double tapping is also not so easy as with a mouse. Compared with these, dragging (drawing) a marker is easier and more direct for the audience,

Smooth and continuous movement of displayed objects (Perlin et al. 1993) seems more important for the shared large display so that the audience can keep their focus of attention without being annoyed by the sudden change of contents.

Consequently, the styles and elements of the desktop GUI must be modified so that they can exploit the IdeaBoard features. However, the modification should be very natural so that users do not feel a big difference.

\section{CONTENTS IN THE VIDEO}

The styles and elements of the the IdeaBoard UI are shown in the video. Several applications which are effectively interacted by the UI are also demonstrated.

This work is being supported by the Advanced Software Enrichment Project of IPA under MITI, Japan.

\section{REFERENCES}

Elrod, S., Bruce, R., Gold, R., Goldberg, D., Halasz, F., Janssen, W., Lee, D., McCall, K., Pedersen, E., Pier, K., Tang, J. and Welch, B. (1992) Liveboard: A Large Interactive Display Supporting Group Meetings, Presentations and Remote Collaboration. Proc. CHI'92, 599-607.

Nakagawa, M., Oguni, T., Yoshino, T. (1997) Human interface and applications on IdeaBoard, to appear in Proc. INTERACT 97.

Pedersen, E.R., McCall, K., Moran, T.P., Halasz, F.G. (1993) Tivoli: An Electronic Whiteboard for Informal Workgroup Meetings, Proc. INTERCHI'93, 391-398.

Perlin, K. and Fox, D. Pad: An Alternative Approach to the Computer Interface, Proc. 1993 ACM SIGGRAPH Conference, 57-64.

Weiser, M. (1991) The Computer for the 21st Century, Scientific American, 94-104.

Weiser, M. (1993) Some Computer Science Issues in Ubiquitous Computing, $C$. $A C M, 36,7,74-84$. 